\title{
Uncovering preferences from patient list data using benefit efficient models
}

\author{
Jan Ubøe, Jostein Lillestøl
}

The Norwegian School of Economics and Business Administration, Bergen, Norway. Email: jan.uboe@nhh.no, jostein.lillestol@nhh.no

Received 11 September 2009; revised 1 June 2010; accepted 5 June 2010.

\begin{abstract}
In this paper it is shown how the benefit efficient patient list model of Ubøe and Lillestøl [1] can be used to infer strength of preferences from patient list data. It is proved that the model allows the construction of unique sets of preferences replicating the observed allocations. To illustrate how this theory can be applied in practice, preferences are uncovered from a small data set, obtained from the Norwegian patient list system.
\end{abstract}

Keywords: Patient Lists; Efficient Welfare; Statistical Distributions

\section{INTRODUCTION}

In the Norwegian patient list system in general practice the patients can be assigned to a general practitioner that agrees to have the main responsibility for his or hers patients. As there are limited numbers of doctors of specific types, e.g. gender, it may happen that a significant number of patients are assigned to doctors of the wrong type, i.e. a type of doctor that they really do not want. This gives rise to several interesting research questions, among them: How can we characterize assignments that conform to reasonable criteria for total benefits to the patient-doctor community, when patients are individually competing for the scarce resource? Which changes in allocation can be expected when the availability of the scarce resource changes, e.g. by increased availability of female doctors? The latter is known to happen in many countries, and was precisely the question asked by the investigators on general practice and community medicine that posed the problem in the first place.

In [1] Ubøe and Lillestøl suggested a new statistical framework for this context, enable to answer questions of this kind, based on the concept of benefit efficiency. The next research challenge was then to see if the model allowed inferences, i.e., to say something about the preference structure based on an observed allocation. This turned out to involve some delicate theoretical problems, among them non-uniqueness, and the purpose of this paper is to present our solution to these problems. As an illustration of the theory we will consider a special case, using patient list data from the Norwegian patient list system in general practice. These data report the registered allocation of male and female patients to male and female doctors. While this is mainly a theoretical paper, the paper also offers some guidelines for practitioners that want to apply this kind of theory to real world data.

The theory of this paper follows from the idea of a benefit efficient allocation, which can be described as follows: Let $A_{1}, A_{2}, \ldots, A_{T}$ denote the sets of actions that agents of type $1,2, \ldots, T$ can choose, let $U_{t}: A_{t} \rightarrow R$ be the real number utilities of each choice, and let $Q_{1}$, $Q_{2}, \ldots, Q_{T}$ be a sequence of probability measures on $A_{1}$, $A_{2}, \ldots, A_{T}$. Then $\boldsymbol{Q}=Q_{1} \times Q_{2} \times \ldots \times Q_{T}$ is called a benefit efficient probability measure if it satisfies the two conditions:

- Larger aggregated utility, i.e. sum of utilities of all agents, of an allocation implies larger probability of that allocation.

- As the number of agents of each type pass to infinity, the numbers of agents making each choice must satisfy a specified set of linear allocation constraints.

Quite surprisingly, there are extremely few probability measures of this type. In fact when utilities and constraints are given, these measures form a one parameter family. In the inverse problem we consider in this paper, the parameter can be set to 1 without loss of generality. Then the resulting allocation will be as given by Formula (1) in the theory section below, where we briefly recall the model construction in [1], and then show how we can obtain unique representations of preferences. To enhance readability, proofs and technical arguments are given in appendices.

The Norwegian patient list system in general practice 
is described in some detail in [1], and we refer to this paper for a review of the system. Note that our modeling framework extends beyond the Norwegian patient list system and it can be understood without any particular knowledge of that system. Note also that our model is completely specified by Formula (1), and no further knowledge of the model will be needed to understand the issues we address in this paper.

In the application section we use our model to infer structure and strength of preferences from observed real world data. These data were collected from an official panel survey of Norwegian living conditions (Levekårsundersøkelsen 2003).

We believe that our basic approach to this problem is novel. It is fundamentally different from the theory of revealed preferences in consumer theory, see e.g. [2], and is not in any way related to the extensive economic literature on the design of matching system in health care, see e.g. [3]. Hence we will not enter into a discussion of other models in this area with a different scope.

\section{METHODS}

The model in [1] can be described briefly as follows: Assume that there are $S$ groups of patients, $T$ types of doctors, and let $P_{t s}$ denote the number of patients in group $s$ that has a doctor of type $t, s=1,2, \ldots, S, t=1$, $2, \ldots, T$.

Patients: We assume that there is a total of $E_{s}$ patients belonging to group $s, s=1,2, \ldots, S$. A patient belonging to group $s$ is assumed to have a utility $U_{t s}$ of having a doctor of type $t, t=1,2, \ldots, T$. It may sometimes happen that a patient prefer to wait for a vacancy of a suitable doctor rather than being assigned to a doctor of a type that the patient dislikes. We let $P_{t(s+S)}$ denote the number of patients of type $s$ waiting for a doctor of type $t$ (not being assigned to any doctor), and let $U_{t(\mathrm{~s}+S)}$ denote the utility of these patients.

- Doctors: Every doctor working within the system is assumed to have a certain list length, i.e., a maximum number of patients that he or she can serve. We assume that there are $D_{t}$ doctors of type $t$, and that these doctors can serve a total of $L_{t}$ patients, i.e., $L_{t}$ is the sum of the list lengths of all doctors of type $t$. Some doctors may have vacancies, and we let $U_{t(2 S+1)}$ denote the utility per vacancy incurred by a doctor of type $t$.

Thus we have defined $P_{t s}$ and $U_{t s}$ for $s=1,2, \ldots, 2 S+$ $1, t=1,2, \ldots, T$, which can then be represented by $\operatorname{Tx}(2 S$ $+1)$ matrices $\boldsymbol{P}$ and $\boldsymbol{U}$.

Utilities for vacancies and for being assigned to the wrong type of doctor may of course be negative, in which case we refer to these numbers as disutilities. Note that the word utility is used in a broad sense as a quantification of strength of preferences. Utilities are hence not necessarily utilities in the von NeumannMorgenstern sense.

Clearly the $\left(E_{1}, \ldots, E_{S}\right)$ patients can be allocated to the $\left(D_{1}, \ldots, D_{T}\right)$ doctors in many different ways. The basic hypothesis in [1], however, is to assume that the system is benefit efficient in the sense that states with large aggregate utility (sum of the utility of all patients and doctors) are more probable than states with smaller total utility. If the system is benefit efficient with a large number of patients in every group, it is possible to prove, see [1], that the allocation will settle at a statistical equilibrium given by the following explicit formula:

$$
\begin{aligned}
& P_{t s}=\left\{\begin{array}{cc}
A_{t} B_{s} \exp \left(U_{t s}\right) & s=1, \ldots, S \\
D_{t} B_{s-S} \exp \left(U_{t s}\right) & s=S+1, \ldots, 2 S \\
A_{\tau} \exp \left(U_{t s}\right) & s=2 S+1
\end{array}\right. \\
& \sum_{s=1}^{S} P_{t s}+P_{t(2 S+1)}=L_{t} \\
& \sum_{t=1}^{T} P_{t s}+P_{t(s+S)}=E_{s}
\end{aligned}
$$

See Appendix 3 on how to compute the balancing factors $A_{1}, A_{2}, \ldots, A_{T}, B_{1}, B_{2}, \ldots, B_{S}$. Note that these allocations must not be confused with the allocation with maximum total utility. In fact, the allocation with maximum total utility can be obtained as special case if one multiplies the utilities in (1) with a constant and let that constant pass to infinity.

The basic problem we want to address in this paper can be formulated as follows: Assume that the system is benefit efficient and that we observe

- The total number of patients in each group, i.e. $E_{s}, s$ $=1, \ldots, S$.

- The total number of doctors of each type, i.e. $D_{t}, t$ $=1, \ldots, T$.

- The total list length of doctors of each type, i.e. $L_{t}, t$ $=1, \ldots, T$.

The final allocation of patients to doctors/waiting lists, i.e. $P_{t s}, s=1, \ldots, 2 S+1, \quad t=1, \ldots, T$.

To what extent do these observations reveal the strength of the preferences

$$
U_{t s}, s=1, \ldots, 2 S+1, t=1, \ldots, T \text { ? }
$$

It is easy to observe that there are always an infinite number of utility matrices leading to the same final allocation. To obtain uniqueness we hence have to impose some additional restrictions on specific utilities and/or the relationships between them. Such restrictions are typically based on prior knowledge of the context and on known empirics, and modeling issues of this kind are discussed in detail in Section 3. In Theorem 2.1 below, we single out one of the infinitely many representations, and refer to the representation in (2) as the canonical choice. This is useful for two main reasons: To 
get insight to the degrees of freedom in modeling, and to provide a basis for numerical calculations.

\subsection{Theorem 2.1}

Assume that an observed patient list distribution $\boldsymbol{P}$ can be replicated by a model that satisfies (1). Then we can find a unique utility matrix $\boldsymbol{U}$ that replicates $\boldsymbol{P}$ on the form (later referred to as (2)):

$\left[\begin{array}{cccccccccc}0 & 0 & 0 & \ldots & 0 & v_{11} & v_{12} & \ldots & v_{1 S} & 0 \\ 0 & u_{11} & u_{12} & \ldots & u_{1(S-1)} & v_{21} & v_{22} & \ldots & v_{1 S} & w_{1} \\ 0 & u_{21} & u_{22} & \ldots & u_{2(S-1)} & \vdots & \vdots & & \vdots & w_{2} \\ \vdots & \vdots & \vdots & \vdots & \vdots & \vdots & \vdots & & \vdots & \vdots \\ 0 & u_{(T-1) 1} & u_{(T-1) 2} & \ldots & u_{(T-1)(S-1)} & v_{T 1} & v_{T 2} & \ldots & v_{T S} & w_{T-1}\end{array}\right]$

PROOF: See Appendix 1.

The zeros in (2) can formally be interpreted as reference points and the corresponding groups as reference groups. Uniqueness is obtained when we specify how much more/less utility the other groups have in comparison to these reference groups. While the representation given by (2) has several favorable properties, results given on this form are quite hard to interpret. Hence it might be profitable to look for other representations offering more transparent interpretations. In general the position of the $S+T$ reference groups can be chosen in several different ways, and an important modeling issue is to specify natural reference groups for the given context.

We can also obtain alternative unique representations by assuming a utility structure with sufficient identities and/or symmetries. Nevertheless, it is convenient to use (2) as a canonical form, both for algorithmic programming and for resolving theoretical issues. One important issue is that of identification, i.e., recovering the parameters of an assumed utility structure from its established canonical form. Equivalent structures are obtained by transformations of $\boldsymbol{U}$ that leave $\boldsymbol{P}$ invariant. These are:

Add/subtract a fixed $T$-dimensional column vector $\boldsymbol{a}$ to all columns of $\boldsymbol{U}$ labeled $s=1, \ldots, S$ and $s=$ $2 S+1$ (i.e. except $s=S+1, \ldots, 2 S$ ).

- Add/subtract a fixed $2 S+1$-dimensional row vector of form $(\boldsymbol{b}, \boldsymbol{b}, 0)$, with $\boldsymbol{b} S$-dimensional, to all rows of $\boldsymbol{U}$.

- Add/subtract a constant $c$ to column $s=2 S+1$ of $\boldsymbol{U}$ and at the same time subtract/add the same constant from all columns $s=S+1, \ldots, 2 S$.

However, the easiest way to check identifiability may be to use the transform given by Formula (11) in Appendix 1 and check the uniqueness of the parameter recovery.

Note that the canonical form, as well as alternative models with the same number of (linear) restrictions, provides perfect fit to the available data. Hence any inference does not lend itself to the usual statistical standard error computations.

\section{RESULTS AND DISCUSSION}

The Norwegian patient list system was introduced in year 2001 and is monitored by the authorities. Data on availability of doctors are made readily available to the public, and some aggregated data on list composition and vacancies are also available for research purposes. Reliable data on doctor preference are not readily available. However, some questions on the combination (gender of respondent, gender of assigned doctor, preferred gender of doctor) were included the official panel survey of Norwegian living conditions (Levekårsundersøkelsen 2003). The questions asked relevant to our study were:

Q1: Do you want to relate to an assigned general practitioner, or do you want to use several general practitioners?

Q2: Do you mind whether your assigned general practitioner is male or female?

Q3: For those who answered "yes" on Q2: Do you want to have a male or a female assigned general practitioner, or do you want to use both a male and a female?

Unfortunately the response rate to the preference questions were low, and more so for males than females. Hence this part of the paper must be considered more as an illustration of the potential offered by the theory, and not so much as an empirical survey in its own right.

With the way of questioning above we were left with the problem of what to do with respondents who wanted both gender of doctors available. Since the system of a single assigned general practitioner was already firmly established with no opportunity of multiple assignments, we decided to split these relatively few respondents equally between the two preferences. The observed counts are shown in Table 1.

In this case $T=2$, with types denoted $\mathrm{M}$ (male doctor) and $\mathrm{F}$ (female doctor), and $S=4$ with groups denoted $\mathrm{mm}, \mathrm{mf}, \mathrm{fm}$ and $\mathrm{ff}$, where the first letter is the gender of the patient and the second letter is the preferred gender of doctor.

A total of 3489 persons, 1736 men and 1753 women, were interviewed in the panel survey. As we can see from Table 1, the responses to the preference questions were very low, and more so for males than females. That partly explains the strong bias we observe in the data, e.g. $22 \%$ men, and we therefore scaled the data to adjust for this. Two issues are taken into account: First

Table 1. Observed counts in each group-original data.

\begin{tabular}{ccccc}
\hline Patient group & $\mathrm{mm}$ & $\mathrm{mf}$ & $\mathrm{fm}$ & $\mathrm{ff}$ \\
\hline M-doctor & 78 & 2 & 32 & 77 \\
F-doctor & 5 & 4 & 2 & 200 \\
\hline
\end{tabular}


we have scaled the data so that there is an equal number of respondents of each sex, second we have scaled the data to get the marginal frequencies in accordance with the approximately known distribution of doctors at the time, namely $70 \%$ male doctors and $30 \%$ female doctors.

The results after these scalings are given in Table 2 per 1000 respondents.

\subsection{Missing Data and Partial Information}

Officially there are no waiting lists, and data on this are hard to get. In Appendix 2, however, we prove that one can infer the correct preferences for the groups in Table 2 even in the case where data on waiting lists and vacancies are missing. The crucial result, Theorem 5.4, states that these preferences are independent of the data for waiting lists and vacancies.

A survey made by the Norwegian Ministry of Health and Care Services (2004) reports a total of 2026 doctors with vacancies, the average number of vacancies being 223. With the reported 4563751 patients served, this gives 99 vacancies per 1000 patients. For illustrative purposes we round this in Table 3 to 100 patients per 1000 served. We have no information on how this is distributed among the gender of doctors. If they are distributed evenly among the genders, the number will be as given in the parentheses in Table 3 . Note that the number of patients who want a doctor of the same gender is higher for males than for females.

\subsection{Inferring Canonical Utilities from Observed Data}

The utility matrix corresponding to the types and groups in Table 3 is

$$
\boldsymbol{U}=\left[\begin{array}{lllllll}
U_{11} & \ldots & U_{14} & U_{15} & \ldots & U_{18} & U_{19} \\
U_{21} & \ldots & U_{24} & U_{25} & \ldots & U_{28} & U_{29}
\end{array}\right]
$$

where the row order is $\mathrm{M}, \mathrm{F}$ and the column order is $\mathrm{mm}$, mf, fm, ff, mm-w, mf-w, fm-w, ff-w, vacancy, where $\mathrm{w}$ indicates a waiting list state. According to Theorem 5.4 in Appendix 2, the missing data in Table 3 can be chosen arbitrarily. Zero entries can be handled, but unless special care is taken such entries lead to serious numerical problems. For simplicity we have carried out all the calculations using the numbers reported in Table 4. We remark, however, that the numbers reported on waiting lists are fictitious but to some extent realistic.

To find replicating utilities, we use the construction described in Proposition 5.3 in Appendix 1. The result is shown in Table 5.

The numbers in parenthesis are based on the fictitious waiting list numbers. Hence the strength of preferences we can infer from the data in Table 2 is given by Table 6 .
If we take the canonical structure as our model, we may infer that that the patients with preference for the scarce gender (female doctors), have high utilities for correct matching compared with the reference groups, and that female patients wanting a male doctor while being assigned to a female doctor tend to have utilities slightly below the reference group zero.

Be aware, however, that this inference may be due to technical artifacts linked to the implicit assumptions caused by a special choice of reference groups. The canonical choice corresponds to an assumption where male patients wanting a male doctor are in effect indifferent to the gender of their doctor, and that may very well be an artificial assumption.

\subsection{Modeling and Further Inference}

We will now look into the modeling aspects of alternative representations. While the utilities reported in Table 5 are the unique utilities on the form (2) that replicates the counts in Table 4, there are several other utility matrices that offer perfect replication. Also, observations of this type are subject to considerable amounts of randomness. Perfect replication is hence relatively unimportant, and models offering less than perfect fit may be superior if they carry more transparent information.

A main issue in this context is to quantify disutilities of incorrect patient/doctor matching. It is straightforward to verify that the solution of (1) is fixed if we add the same constant to all utilities within the same group, i.e., our model is sensitive to utility differences but does not depend on the general level of utility. Without loss of generality we can hence assume that all utilities for correct patient/doctor matching are equal to zero. These assumptions lead us to consider utility matrices on the form:

$$
\boldsymbol{U}=\left[\begin{array}{ccccccccc}
0 & a_{2} & 0 & a_{4} & b_{11} & b_{12} & b_{13} & b_{14} & c_{1} \\
a_{1} & 0 & a_{3} & 0 & b_{21} & b_{22} & b_{23} & b_{24} & c_{2}
\end{array}\right]
$$

Table 2. Adjusted data from Table 1.

\begin{tabular}{lcccc}
\hline Patient group & $\mathrm{mm}$ & $\mathrm{mf}$ & $\mathrm{fm}$ & $\mathrm{ff}$ \\
\hline M-doctor & 455 & 12 & 69 & 164 \\
F-doctor & 19 & 14 & 2 & 265 \\
Total & 474 & 26 & 71 & 429 \\
\hline
\end{tabular}

Table 3. Scaled counts in each group per 1000 patients served.

\begin{tabular}{lccccccccc}
\hline Patient group & mm & $\mathrm{mf}$ & $\mathrm{fm}$ & $\mathrm{ff}$ & $\mathrm{mm}-\mathrm{w}$ & $\mathrm{mf}-\mathrm{w}$ & $\mathrm{fm}-\mathrm{w}$ & $\mathrm{ff}-\mathrm{w}$ & $\mathrm{vac}$ \\
\hline M-doctor & 455 & 12 & 69 & 164 & - & - & - & - & $(70)$ \\
F-doctor & 19 & 14 & 2 & 265 & - & - & - & - & $(30)$ \\
\hline Total & 474 & 26 & 71 & 429 & - & - & - & - & 100 \\
\hline
\end{tabular}


Table 4. Scaled counts with artificial waiting list data.

\begin{tabular}{lccccccccc}
\hline Patient group & $\mathrm{mm}$ & $\mathrm{mf}$ & $\mathrm{fm}$ & $\mathrm{ff}$ & $\mathrm{mm}-\mathrm{w}$ & $\mathrm{mf}-\mathrm{w}$ & $\mathrm{fm}-\mathrm{w}$ & $\mathrm{ff}-\mathrm{w}$ & vac \\
\hline M-doctor & 455 & 12 & 69 & 164 & $(8)$ & $(4)$ & $(9)$ & $(1)$ & $(70)$ \\
F-doctor & 19 & 14 & 2 & 265 & $(2)$ & $(6)$ & $(4)$ & $(6)$ & $(30)$ \\
\hline Total & 474 & 26 & 71 & 429 & $(10)$ & $(10)$ & $(13)$ & $(7)$ & 100 \\
\hline
\end{tabular}

Table 5. Canonical utilities acording to (2) with Table 4 data.

\begin{tabular}{cccccccccc}
\hline $\mathrm{P}$ & $\mathrm{mm}$ & $\mathrm{Mf}$ & $\mathrm{fm}$ & $\mathrm{ff}$ & $\mathrm{mm}-\mathrm{w}$ & $\mathrm{mf}-\mathrm{w}$ & $\mathrm{fm}-\mathrm{w}$ & $\mathrm{ff}-\mathrm{w}$ & $\mathrm{vac}$ \\
\hline $\mathrm{M}$ & 0 & 0 & 0 & 0 & $(-7.7)$ & $(-4.7)$ & $(-5.7)$ & $(-8.7)$ & 0 \\
$\mathrm{~F}$ & 0 & 3.3 & -0.4 & 3.7 & $(-8.2)$ & $(-3.7)$ & $(-5.6)$ & $(-6.1)$ & $(2.3)$ \\
\hline
\end{tabular}

Table 6. Canonical utilities (2) inferred from Table 2.

\begin{tabular}{cccccccccc}
\hline Patient group & $\mathrm{mm}$ & $\mathrm{mf}$ & $\mathrm{fm}$ & $\mathrm{ff}$ & $\begin{array}{c}\mathrm{mm} \\
-\mathrm{w}\end{array}$ & $\begin{array}{c}\mathrm{mf} \\
-\mathrm{w}\end{array}$ & $\begin{array}{c}\mathrm{fm} \\
-\mathrm{w}\end{array}$ & $\mathrm{ff}-\mathrm{w}$ & $\mathrm{vac}$ \\
\hline M-doctor & 0 & 0 & 0 & 0 & - & - & - & - & 0 \\
F-doctor & 0 & 3.33 & -0.37 & 3.66 & - & - & - & - & - \\
\hline
\end{tabular}

Here $a_{1}, a_{2}, a_{3}, a_{4}$ are the disutilities of incorrect patient/doctor matching. The question is now if it is possible to find utility matrices of the form (4) replicating the counts in Table 4 . The transformation defined by Formula (11) in Appendix 1 transforms any utility matrix $\boldsymbol{U}$ to an equivalent matrix $\boldsymbol{U}$ on the form (2).

Equivalent means that the two matrices produce exactly the same counts when they are used in (1). Using (11) on the matrix in (4) we obtain Formula (5). We see that we do not have identifiability, unless we add restrictions. Nevertheless it is possible to infer some non-trivial relationships. If the utility matrix in (5) equals the matrix specified in Table 5 or $\mathbf{6}$, we get the equations

$$
\begin{aligned}
-a_{2}-a_{1} & =3.33 \\
a_{3}-a_{1} & =-0.37 \\
-a_{4}-a_{1} & =3.66
\end{aligned}
$$

If we eliminate $a_{1}$ from the first and the third equation and rearrange the terms, we see that:

$$
\begin{aligned}
& a_{3}=a_{1}-0.37 \\
& a_{4}=a_{2}-0.33
\end{aligned}
$$

By assumption, the utilities for correct patient/ doctor matching are all equal to zero, and hence (by context) utilities for incorrect matching must all be negative (if they are not, agents must have been allocated to wrong groups). In (7) $a_{1}, a_{2}$ are the disutilities of incorrect matching for men, and $a_{3}, a_{4}$ are the disutilities of incorrect matching for women. From (7) we can draw the conclusion that disutilities of incorrect matching are larger for women.

If we go one step further, we can introduce the additional assumptions: $a_{1}=a_{2}=a_{m}$ and $a_{3}=a_{4}=a_{f}$, i.e. that all men have the same disutility of incorrect matching, and all women have the same disutility of incorrect matching. In this case the system is overidentified, and perfect fit cannot be obtained. As mentioned above, perfect fit is relatively unimportant, however, and we can instead search for the best possible fit using utility matrices on the form

$$
\boldsymbol{U}=\left[\begin{array}{ccccccccc}
0 & a_{m} & 0 & a_{f} & b_{11} & b_{12} & b_{13} & b_{14} & c_{1} \\
a_{m} & 0 & a_{f} & 0 & b_{21} & b_{22} & b_{23} & b_{24} & c_{2}
\end{array}\right]
$$

The best replication, in the sense that we get an approximate solution to (6), is then

$$
a_{m}=-1.66 \quad a_{f}=-2.02
$$

In this case all the relevant parameters are identified by our assumptions, and we maintain the conclusion from (7), i.e., that disutilities of incorrect matching are larger for women. It is somewhat surprising that this rather crude approach produces the near perfect fit shown in Table 7 (to be compared with observations in Table 2).

If we want to infer disutilities for vacancies or for being on waiting lists, we would have needed data for the allocation of these groups. Such data are missing. Nevertheless we will look into some general issues connected to these data.

If we compare the lower right corners in Table 5 and Eq.5, we obtain the equation $c_{2}-c_{1}-a_{1}=$ (2.33). This equation could in principle offer an alternative line to identification. If we assume that the disutilities for vacancies are equal for both gender of doctors, i.e., that $c_{1}=c_{2}$, we could infer the value $a_{1}=(-2.33)$. This value could then have been inserted in (6) to identify the remaining disutilities in (6).

To proceed one step further, assume that all disutilities for being on waiting lists are equal, i.e., that $b_{i j}=b$ for all $i=1,2$ and $j=1, \ldots, 4$, and that the disutilities for vacancies are equal for both gender of doctors, i.e., that $c_{1}=c_{2}=c$. Then we could try to find the best possible fit using utility matrices on the form

$$
\boldsymbol{U}=\left[\begin{array}{ccccccccc}
0 & a_{m} & 0 & a_{f} & b & b & b & b & c \\
a_{m} & 0 & a_{f} & 0 & b & b & b & b & c
\end{array}\right]
$$

When modeling the utility structure with sufficient assumptions to get excess degrees of freedom, we essen-

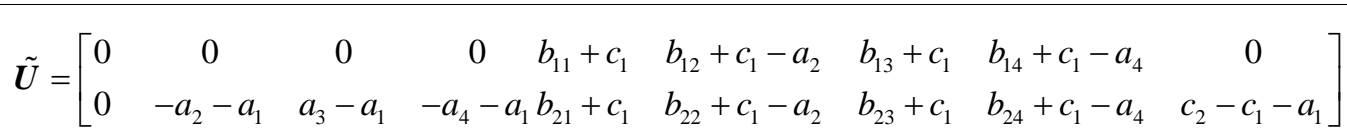


tially have an estimation problem with an opportunity to judge the sampling error. It may then be helpful to bring the problem within the framework of conventional contingency table theory. In our application this may be done by lifting the $2 \times 4$-block of waiting list patients up as a second layer on top of the $2 \times 4$-block of assigned patients. On top of the block of vacancies we add a block of structural zeros. Thus we have a three-way table of size $2 \times 5 \times$ 2 with the correct marginal features. By taking logarithms in the representation (1), we get a linear expression for the log-counts. Our modeling assumptions then give rise to a log-linear model with a specific parametric structure to be estimated, and for which readily available and applicable general theory exists. In the model (10), we have 4 parameters $a_{m}, a_{f}, b$ and $c$, in addition to the 8 scaling constants $A_{i}, D_{i}, i=1,2$ and $B_{j}, j=1,2,3,4$. These parameters may then be estimated by maximum-likelihood principle, for which asymptotic theory is available and provides approximate standard errors of estimates, see, e.g. Bishop et al (1975).

Remark. To estimate the parameters in (10) we would have needed to make use of the artificial data in Table 4. These data were not available and were included for illustration only. To some extent the method suggested for (10) is also relevant for the analysis of (8). As shown in Table 7, a simplistic approach already produces near perfect fit, and alternative/more refined methods would not change this. For these reasons we will not pursue this further.

\section{CONCLUSIONS}

In [1] Ubøe and Lillestøl proposed a new type of statistical model to study the allocation of groups of patients to different types of doctors according to given preferences. This paper clarifies the inverse problem of how to identify preferences based a given allocation.

As an illustration of the theory we have applied the model to patient list data from the Norwegian patient list system in general practice. However, this type of model can be used to infer preferences from much more refined systems than the one we have studied here. We only

Table 7. Counts using $a_{m}=-1.66, a_{f}=-2.02$.

\begin{tabular}{lcccc}
\hline Patient group & $\mathrm{mm}$ & $\mathrm{mf}$ & $\mathrm{fm}$ & $\mathrm{ff}$ \\
\hline M-doctor & 455 & 12 & 69 & 163 \\
F-doctor & 19 & 14 & 2 & 266 \\
Total & 474 & 26 & 71 & 429 \\
\hline
\end{tabular}

made use of two types of doctors and four groups of patients, while the model allows arbitrary many types of doctors and arbitrary many groups of patients.

The revealed preferences from the Norwegian patient list data turned out to be very reasonable, and mostly in accordance with prior beliefs. The main empirical finding that disutilities for incorrect matching are larger for women than for men appears to be a relatively robust conclusion that can be inferred from different model formulations.

Despite the weakness of our data, they may give some backing for the health authorities, e.g., when asking questions like: What changes are likely to happen when the fraction of female doctors is on the rise? This may be answered by using the model in the forward manner, as described in [1], and in more detail in [5]. Revealed disutilities are then used as input, representing the current preference status. It would clearly be of interest to have periodic updates on patient allocations and preferences to investigate the stability of disutilities.

\section{ACKNOWLEDGEMENTS}

The authors wish to thank Yngve Rønsen MD who proposed the patient list problem and Gry Henriksen at the Norwegian Social Science Services (NSD) for making the patient list data available to us.

\section{REFERENCES}

[1] Ubøe, J. and Lillestøl, J. (2007) Benefit efficient statistical distributions on patient lists. Journal of Health Economics, 26(4), 800-820.

[2] Varian, H.R. (2006) Revealed preferences. in Szenberg, M., ed., Samuelsonian Economics and the 21st Century, Oxford University Press, 99-115.

[3] Roth, A.E. and Peranson, E. (1999) The redesign of the matching market for American physicians: Some engineering aspects of economic design. The American Economic Review, 89(4), 748-780.

[4] Bishop, Y.M.M. Fienberg, S.E. and Holland, P.W. (1975) Discrete multivariate analysis: Theory and practice. The MIT Press, Cambridge Mass.

[5] Lillestøl, J., Ubøe, J., Rønsen, Y. and Hjortdahl, P. (2007) Patient allocations according to circumstances and preferences. Discussion paper, Norwegian School of Economics and Business Administration, Bergen.

[6] Bregman, L.M. (1967) The relaxation method of finding the common point of convex sets and its application to the solution of problems in convex programming. USSR Computational Mathematics and Mathematical Physics, 7, 200-217. 


\section{APPENDICES}

\section{Appendix 1: Proof of Theorem 2.1}

Consider the following matrix transformation

$$
\tilde{U}_{t s}=\left\{\begin{array}{cc}
U_{t s}-U_{t 1}-U_{1 s}+U_{11} & s=1, \ldots, S \\
U_{t s}-U_{1(s-S)}+U_{1(2 S+1)} & s=S+1, \ldots, 2 S \\
U_{t s}-U_{t 1}-U_{1(2 S+1)}+U_{11} & s=2 S+1
\end{array}\right.
$$

\section{LEMMA 5.1}

Let $\boldsymbol{U}=\left\{U_{t s}\right\}$ be given, let $\tilde{U}=\left\{\tilde{U}_{t s}\right\}$ be defined by (11) and let $P$ and $\tilde{P}$ denote the corresponding distributions of patients in (1) when we use $\boldsymbol{U}$ and $\tilde{U}$, respectively. Then $P=\tilde{P}$.

PROOF

Let $A_{1}, \ldots, A_{T}, B_{1}, \ldots, B_{S}$ denote the balancing factors solving (1) when we use $\boldsymbol{U}$, and define

$$
\begin{aligned}
& \tilde{A}_{t}=A_{t} \exp \left(U_{t 1}+U_{1(2 S+1)}-U_{11}\right) t=1, \ldots, T \\
& \tilde{B}_{s}=B_{s} \exp \left(U_{1 s}-U_{1(2 S+1)}\right) s=1, \ldots, S \\
& \text { If } s=1, \ldots, s \text { we get } \\
& \tilde{A}_{t} \tilde{B}_{s} \exp \left(\tilde{U}_{t s}\right)=A_{t} B_{s} \exp \left(U_{t s}\right) \\
& D_{t} \tilde{B}_{s-S} \exp \left(\tilde{U}_{t s}\right)=D_{t} B_{s-S} \exp \left(U_{1(s-S)}-U_{1(2 S+1)}\right) \\
& \times \quad \exp \left(U_{t s}-U_{1(s-S)}+U_{1(2 S+1)}\right) \\
& =\quad D_{t} B_{s-S} \exp \left(U_{t s}\right) \\
& \tilde{A}_{t} \exp \left(\tilde{U}_{t s}\right)=A_{t} \exp \left(U_{t 1}+U_{1(2 S+1)}-U_{11}\right) \\
& \times \exp \left(U_{t s}-U_{t 1}-U_{1(2 S+1)}+U_{11}\right) \\
& =\quad A_{t} \exp \left(U_{t s}\right)
\end{aligned}
$$

which proves the lemma.

\section{PROPOSITION 5.2}

Let $\boldsymbol{U}^{(1)}$ and $\boldsymbol{U}^{(2)}$ denote two utility matrices, and assume that $\boldsymbol{P}^{(1)}=\boldsymbol{P}^{(2)}$ in (1). Using the transformation in (11) we have $\tilde{U}^{(1)}=\tilde{U}^{(2)}$.

PROOF

We have to prove that the balancing factors must be equal, and then it follows from (1) that all the utilities must be equal as well. It follows from Lemma 2.1 that $\tilde{\boldsymbol{P}}^{(1)}=\tilde{\boldsymbol{P}}^{(2)}$, and observe from (11) that $\tilde{U}_{1(2 S+1)}^{(1)}=$ $\tilde{U}_{1(2 S+1)}^{(2)}=0$ by definition. Since

$$
\tilde{A}_{1}^{(1)} \exp \left(\tilde{U}_{1(2 S+1)}^{(1)}\right)=\tilde{A}_{1}^{(2)} \exp \left(\tilde{U}_{1(2 S+1)}^{(2)}\right)
$$

it follows that $\tilde{A}_{1}^{(1)}=\tilde{A}_{1}^{(2)}$. Now put $t=1$ and $s=1, \ldots$, $S$, and observe from (11) that $\tilde{U}_{1 \mathrm{~s}}^{(1)}=\tilde{U}_{1 \mathrm{~s}}^{(2)}=0$ by definition. Hence from (1) we get

$$
\tilde{A}_{1}^{(1)} \tilde{B}_{\mathrm{s}}^{(1)} \exp \left(\tilde{U}_{1 s}^{(1)}\right)=\tilde{A}_{1}^{(2)} \tilde{B}_{\mathrm{s}}^{(2)} \exp \left(\tilde{U}_{1 \mathrm{~s}}^{(2)}\right)
$$

Since $\tilde{A}_{1}^{(1)}=\tilde{A}_{1}^{(2)}$ it follows that $\tilde{B}_{s}^{(1)}=\tilde{B}_{s}^{(2)}$ for all $s$ $=1, \ldots, S$. We then put $s=1$ and $t=1, \ldots, T$, and observe from (11) that $\tilde{U}_{t 1}^{(1)}=\tilde{U}_{t 1}^{(2)}=0$ by definition. From (1) again we get

$$
\tilde{A}_{t}^{(1)} \tilde{B}_{1}^{(1)} \exp \left(\tilde{U}_{t 1}^{(1)}\right)=\tilde{A}_{t}^{(2)} \tilde{B}_{1}^{(2)} \exp \left(\tilde{U}_{t 1}^{(2)}\right)
$$

Since $\tilde{B}_{1}^{(1)}=\tilde{B}_{1}^{(2)}$ it follows that $\tilde{A}_{t}^{(1)}=\tilde{A}_{t}^{(2)}$ for all $t=1, \ldots, T$. We have hence proved the proposition.

Proof of Theorem 2.1

By assumption we can find a matrix $\boldsymbol{U}$ that replicates $P$. According to Lemma $5.1 \tilde{U}$ also replicates $P$. By construction $\tilde{U}$ is on the special format given by (2). Hence there exists a matrix on the form (2) that replicates $P$. Conversely if a matrix is of the form given by (2), it does not change when we apply the transformation given by (11). Uniqueness then follows from Proposition 5.2.

\section{PROPOSITION 5.3}

Let $\left\{P_{t s}^{\text {obs }}\right\}$ be the observed numbers on the patient lists. The replicating matrix in Theorem 2.1 can then be constructed as follows. Put:

$$
U_{t s}=\left\{\begin{array}{ccc}
\ln \left(P_{t s}^{o b s}\right) & \text { if } & s=1, \ldots, S \\
\ln \left(P_{t s}^{o b s} / D_{t}\right) & \text { if } & s=S+1, \ldots, 2 S \\
\ln \left(P_{t s}^{o b s}\right) & \text { if } & s=2 S+1
\end{array}\right.
$$

If we put $A_{t}=1, B_{s}=1$ in (1), it is easy to verify that the model in (1) replicates the observed pattern. The unique replicating matrix in Theorem 2.1 can then be found applying the transformation in (11) to the utilities in (19).

\section{Appendix 2: Inference under Partial Information}

Assume that we know the number of patients on the patient lists and the number of vacancies, but do not know how many patients that are waiting for a vacancy. Is it then possible to infer the strength of preferences of the patients on the patient lists? The answer is yes, and this can be demonstrated as follows:

Assume that $P_{t s}^{(0)} \quad s=1, \ldots, S, t=1, \ldots, T$ is given, and let $P_{t s}^{(1)}$ and $P_{t s}^{(2)}$ be arbitrary numbers for $s=S+$ $1, \ldots, 2 S+1, t=1, \ldots, T$. Define the following aggregated quantities 


$$
\begin{gathered}
L_{t}^{(0)}=\sum_{S=1}^{S} P_{t s}^{(0)}, E_{t}^{(0)}=\sum_{T=1}^{T} P_{t s}^{(0)}, \\
L_{t}^{(i)}=\sum_{S=S+1}^{2 S+1} P_{t s}^{(i)}, E_{s}^{(i)}=\sum_{T=1}^{T} P_{t s}^{(i)}, i=1,2
\end{gathered}
$$

\section{THEOREM 5.4}

For $i=1,2$ put $L_{t}=L_{t}^{(0)}+L_{t}^{(i)}, E_{s}=E_{s}^{(0)}+E_{s}^{(i)}$ and find a unique matrix $U^{(i)}$ of the form (2) such that the system given by (1) replicates the numbers

$$
P_{t s}= \begin{cases}P_{t s}^{(0)} & \text { if } \quad s=1, \ldots, S, t=1, \ldots, T \\ P_{t s}^{(i)} & \text { if } \quad s=S+1, \ldots, 2 S+1\end{cases}
$$

If $K=P_{1(2 S+1)}^{(2)} / P_{1(2 S+1)}^{(1)}$ then the two utility matrices $\boldsymbol{U}^{(1)}$ and $\boldsymbol{U}^{(2)}$ are connected through the formula (here referred to as Eq.21):

$$
U_{t s}^{(2)}=\left\{\begin{array}{cc}
U_{t s}^{(1)} & s=1, \ldots, S, t=1, \ldots, T \\
U_{t s}^{(1)}+\ln \left(\frac{P_{t s}^{(2)}}{P_{t s}^{(1)}}\right)+\ln (K) & s=S+1, \ldots, 2 S, t=1, \ldots, T \\
U_{t s}^{(1)}+\ln \left(\frac{P_{t s}^{(2)}}{P_{t s}^{(1)}}\right)-\ln (K) & s=2 S+1, t=1, \ldots, T
\end{array}\right.
$$

PROOF

Define a new utility matrix $\tilde{\boldsymbol{U}}$ by the right hand side of (21) and let $A_{t}^{(1)}, t=1, \ldots, T$ and $B_{s}^{(1)} s=1, \ldots, S$ denote the balancing factors solving (1) using the replicating utilities $\boldsymbol{U}^{(1)}$ Now put $A_{t}^{(2)}=A_{t}^{(1)} \cdot K$ and

$$
\begin{aligned}
& B_{s}^{(2)}=B_{s}^{(1)} / K \text {. } \\
& \text { If } s=1, \ldots, S, t=1, \ldots, T \text { we get by (1) } \\
& A_{t}^{(2)} B_{s}^{(2)} \exp \left(\tilde{U}_{t s}\right)=A_{t}^{(1)} \cdot K \cdot B_{s}^{(1)} / \operatorname{Kexp}\left(U_{t s}^{(1)}\right) \\
& =A_{t}^{(1)} B_{s}^{(1)} \exp \left(U_{t s}^{(1)}\right)=P_{t s}^{(0)} \\
& \text { If } s=S+1, \ldots, 2 S, t=1, \ldots, T \text { we get by (1) } \\
& D_{t} B_{s-S}^{(2)} \exp \left(\tilde{U}_{t s}\right) \\
& =D_{t} B_{s-S}^{(1)} / K \operatorname{Kexp}\left(U_{t s}^{(1)}+\ln \left(\frac{P_{t s}^{(2)}}{P_{t s}^{(1)}}\right)+\ln (K)\right) \\
& =\quad P_{t s}^{(1)} \cdot \frac{P_{t s}^{(2)}}{P_{t s}^{(1)}}=P_{t s}^{(2)} \ln (K) \\
& \text { If } s=2 S+1, t=1, \ldots, T \text { we get by (1) } \\
& A_{t}^{(2)} \exp \left(\tilde{U}_{t s}\right)=A_{t}^{(1)} \cdot \operatorname{Kexp}\left(U_{t s}^{(1)}+\ln \left(\frac{P_{t s}^{(2)}}{P_{t s}^{(1)}}\right)-\ln (K)\right) \\
& =P_{t s}^{(1)} \cdot \frac{P_{t s}^{(2)}}{P_{t s}^{(1)}}=P_{t s}^{(2)}
\end{aligned}
$$

The marginal constraints are automatically satisfied when the model replicates each entry in the matrix. Note that

$$
\tilde{U}_{1(2 S+1)}=U_{1 s}^{(1)}+\ln \left(\frac{P_{1(2 S+1)}^{(2)}}{P_{1(2 S+1)}^{(1)}}\right)-\ln (K)=U_{t s}^{(1)}=0
$$

and that if $s=1, \ldots, S$, then $\tilde{U}_{t s}=U_{t s}$. This proves that $\tilde{\boldsymbol{U}}$ is of the form (2). Hence if we put $\boldsymbol{U}^{(2)}=\tilde{\boldsymbol{U}}$ this matrix is the unique matrix on the form (2) that replicates the system in (20) when $i=2$.

As we can see from Theorem 6.1, the utilities $U_{t s} \mathrm{~s}$ $=1, \ldots, S, t=1, \ldots, T$ do not depend on the values of $P_{t s}$ for $s=S+1, \ldots, 2 S+1, t=1, \ldots, T$. Hence we have the following corollary:

\section{COROLLARY}

Assume that $P_{t s} s=1, \ldots, S, t=1, \ldots, T$ are known, while data on $P_{t s}$ for $s=S+1, \ldots, 2 S+1, t=1, \ldots, T$ are missing. If we choose $P_{t s}>0$ for $s=S+1, \ldots, 2 S$ $+1, t=1, \ldots, T$ arbitrarily, we can still infer the correct values on $U_{t s}$ for $s=1, \ldots, S, t=1, \ldots, T$.

Remark. From the bottom line in Formula (21) we see that we can also obtain strength of preferences for vacancies in cases where information on the number of patients waiting for vacancies is missing. Clearly, however, it is impossible to infer strength of preferences for groups of patients waiting for vacancies unless we have data for these groups.

\section{Appendix 3: Numerical Methods}

How to find a numerical solution to (1) when utilities $\boldsymbol{U}$ and marginal constraints $\boldsymbol{L}$ and $\boldsymbol{E}$ are given? We need to find numerical values for the $S+T$ balancing factors $A_{1}, \ldots, A_{T}, B_{1}, \ldots, B_{S}$. This is done as follows:

Initially we put all the balancing factors equal to 1 . Then for $t=1, \ldots, T$ we update $A_{t}$ using

$$
A_{t}=\frac{L_{t}}{\sum_{s=1}^{S} B_{s} \exp \left(U_{t s}\right)+P_{t(2 S+1)}}
$$

Once these are updated, then for $s=1, \ldots, \mathrm{S}$ we update $B_{s}$ using

$$
B_{s}=\frac{E_{s}}{\sum_{t=1}^{T}\left(A_{t} \exp \left(U_{t s}\right)+D_{t} \exp \left(U_{t(s+S)}\right)\right)}
$$

We then repeat the updates in (22) and (23) until the system settles. The algorithm is a variant of the Bregman balancing algorithm, see Bregman (1967). Like the standard Bregman algorithm this algorithm is surprisingly efficient, and solves large systems in a very short time. 